\title{
Impact of cow strain and concentrate supplementation on grazing behaviour, milk yield and metabolic state of dairy cows in an organic pasture-based feeding system
}

\author{
C. Heublein ${ }^{1,2}$, F. Dohme-Meier ${ }^{1}$, K.-H. Südekum ${ }^{2}$, R. M. Bruckmaier ${ }^{3}$, S. Thanner ${ }^{1}$ and \\ F. Schori ${ }^{1+}$
}

${ }^{1}$ Agroscope, Institute for Livestock Sciences (ILS), Tioleyre 4, 1725 Posieux, Switzerland; ${ }^{2}$ Institute of Animal Science, University of Bonn, Endenicher Allee 15, 53115 Bonn, Germany; ${ }^{3}$ Veterinary Physiology, Vetsuisse Faculty, University of Berne, Bremgartenstrasse 109a, 3001 Berne, Switzerland

\begin{abstract}
As ruminants are able to digest fibre efficiently and assuming that competition for feed $v$. food use would intensify in the future, cereals and other field crops should primarily be destined to cover the dietary needs of humans and monogastric animals such as poultry and pigs. Farming systems with a reduced or absent concentrate supplementation, as postulated by organic agriculture associations, require adapted dairy cows. The aim of this experiment was to examine the impact of concentrate supplementation on milk production, grazing and rumination behaviour, feed intake, physical activity and blood traits with two Holstein-Friesian cow strains and to conclude the consequences for sustainable and organic farming. The experiment was a cross-over study and took place on an organic farm in Switzerland. In all, 12 Swiss Holstein-Friesian (HCH) cows and 12 New Zealand Holstein-Friesian (HNZ) cows, which were paired according to lactation number, days in milk and age for primiparous cows, were used. All cows grazed full time and were supplemented either with $6 \mathrm{~kg} /$ day of a commercial, organic cereal-grain mix or received no supplement. After an adaptation period of 21 days, a measurement period of 7 days followed, where milk yield and composition, pasture dry matter intake estimated with the $\mathrm{n}$-alkane double-indicator technique, physical activity based on pedometer measurements, grazing behaviour recorded by automatic jaw movement recorder and blood samples were investigated. Non-supplemented cows had a lower milk yield and supplemented HCH cows produced more milk than supplemented HNZ cows. Grazing time and physical activity were greater for non-supplemented cows. Supplementation had no effect on rumination behaviour, but HNZ cows spent longer ruminating compared with $\mathrm{HCH}$ cows. Pasture dry matter intake decreased with the concentrate supplementation. Results of blood analysis did not indicate a strong negative energy balance for either non-supplemented or supplemented cows. Minor differences between cow strains in this short-term study indicated that both cow strains are equally suited for an organic pasturebased production system with no concentrate supplementation. Many factors such as milk yield potential, animal welfare and health, efficiency, grazing behaviour and social aspects influence the decision to supplement grazing dairy cows with concentrates.
\end{abstract}

Keywords: concentrate supplementation, organic farming, dairy cow, Holstein, pasture

\section{Implications}

In the future, competition between feed and food will increase. Ruminants like dairy cows are able to digest forage fibre efficiently. As concentrate supplementation is limited in organic dairy farming, restrictions may cause health problems as energy requirements for high-yielding dairy cows may not be met from forage-only rations. The aim of this study was to verify how supplementation changes the behaviour and production of grazing dairy cows under organic conditions. The two Holstein cow strains investigated

\footnotetext{
${ }^{\dagger}$ E-mail: fredy.schori@agroscope.admin.ch
}

in this short-term study are equally suited for an organic, pasture-based farming system with no concentrate supplementation.

\section{Introduction}

In Switzerland, the milk yield per cow has increased steadily over the last years (Bundesamt für Landwirtschaft, 2015), as has the cows' demand for nutrients and energy. This leads to the question of whether high-yielding dairy cows are still able to meet their energy requirements for efficient milk production on pasture-only diets. Farming systems with a reduced or 
absent concentrate supplementation, as postulated by organic agriculture associations, require adapted dairy cows. Studies suggest that high producing Holstein-Friesian (HF) cows are less suited for grazing systems, particularly under high stocking rates and seasonal calving systems and without concentrate supplementation (Kolver and Muller, 1998). In New Zealand, HF cows are bred for efficient pasture use with very little concentrate supplementation and seasonal calving (Washburn and Mullen, 2014). They differ in BW, average body condition score (BCS) and milk yield from other HF cow strains (Piccand et al., 2013) and have a lower milk production response to concentrate supplementation compared with HF cow strains with a high genetic merit for milk production (Horan et al., 2005). In addition, they may be able to use pasture more efficiently for milk production (Macdonald et al., 2008) and therefore may be better suited for organic milk production systems. According to Penning and Rutter (2004), behaviour exhibited by animals is an indication of the relationship between their internal state (e.g. nutritional requirements, health) and their environment (e.g. sward state, supplementation and climate). Therefore, grazing behaviour such as grazing duration, herbage intake and intake rate may provide some evidence about well-suited dairy cows and should be examined in detail, as the efficient use of pasture is a priority. According to the knowledge of the authors, this is the first study that investigated the effect of concentrate supplementation on the eating and rumination behaviour, metabolic states and milk production of grazing dairy cows under organic farming conditions.

The aim of this study was to examine the milk yield and milk composition, grazing and rumination behaviour, physical activity and metabolic profile of grazing cows with and without concentrate supplementation under organic conditions. Furthermore, this study aimed to identify differences between two HF cow strains, especially on grazing and rumination behaviour, which could indicate a better suitability for pasture-based milk production systems with restricted concentrate supplementation for organic farming.

\section{Material and methods}

\section{Animals and experimental design}

The experiment was a $2 \times 2$ cross-over study with two concentrate levels and two cow strains. All experimental procedures were in accordance with the Swiss guidelines for animal welfare and were approved (no. 2012_10_FR) by the Animal Care Committee of the Canton of Fribourg, Switzerland. Before selecting the cows for the experiment, a medical examination was performed. The experiment consisted of two measurement periods, each consisting of a 21-day adaption period and a 7-day measurement period. For the flow of work and equipment reasons, the cows were equally divided into two consecutive data collection periods of 7 days/measurement period. The experiment took place on the organic farm 'Ferme École de Sorens', located $824 \mathrm{~m}$ above sea level in Sorens, Switzerland.
A total of $24 \mathrm{HF}$ cows, including 12 Swiss Holstein-Friesian (HCH) cows and $12 \mathrm{HF}$ cows of New Zealand origin (HNZ), were used for the experiment. In all, 14 of them were multiparous and 10 were primiparous. Matched pairs of $\mathrm{HCH}$ and HNZ cows were formed according to the number of lactation, days in milk (DIM) and age for primiparous cows. The average economic breeding value (ISEL; Swiss Holstein Breeding Association, Posieux, Switzerland) was 985 (SD 74) for $\mathrm{HCH}$ cows. The average economic breeding value for HNZ cows was 806 (SD 70), but this excluded four animals as no breeding value was available.

At the start of the first data collection period, $\mathrm{HCH}$ cows had an average number of lactations of 2.3 (SD 1.6), had been 109 (SD 17.9) DIM, had an average BW of 609 (SD 90.1) $\mathrm{kg}$, a BCS of 2.4 (SD 0.28) and were producing 27.6 (SD $3.77) \mathrm{kg}$ milk/day. The HNZ cows had an average number of lactations of 2.7 (SD 2.0), had been 114 (SD 16.7) DIM, had an average BW of 560 (SD 72.3) kg, a BCS of 2.9 (SD 0.24) and were producing 24.1 (SD 5.27) kg milk/day.

\section{Grazing management, concentrate supplementation and weather conditions}

The experiment was carried out in a rotational grazing system from 7 May to 8 July 2012. All 24 experimental cows were managed as a single group separated from the rest of the lactating herd and grazed on pasture from 0800 to $1400 \mathrm{~h}$ and from 1800 to $0430 \mathrm{~h}$ the following morning. Meanwhile, cows were milked and housed in a free-stall barn. Paddocks were rotationally grazed for 2 to 5 days based on decision rules considering sward height with a reference of $130-\mathrm{mm}$ pre-grazing equivalent to an herbage mass of $\sim 1000 \mathrm{~kg}$ dry matter (DM)/ha above $50 \mathrm{~mm}$ until a post-grazing sward surface height of $50 \mathrm{~mm}$ from ground level. The sward surface height was measured with a pasture meter (C-Dax pasture meter; C-Dax Ltd, Turitea, New Zealand) before cows entered a new paddock and after leaving the paddock. The average pre-grazing sward height was 129 (SD 16.4; $n=5$ ) mm, corresponding to 1024 (SD 209.3) kg DM/ha above $50 \mathrm{~mm}$ in the first measurement period and $122($ SD 11.2; $n=6) \mathrm{mm}$, corresponding to 941 (SD 143.8) kg DM/ha for the second measurement period. The average post-grazing sward surface height was 57 (SD 6.6; $n=5) \mathrm{mm}$ in the first measurement period and 71 (SD 19.2; $n=6$ ) $\mathrm{mm}$ in the second measurement period. Herbage mass above $50 \mathrm{~mm}$ (kg DM/ha) was calculated according to: $-624.5+12.8 \times$ sward height $(\mathrm{mm})$. This regression was calibrated for the pastures of the organic farm 'Ferme École de Sorens' $\left(R^{2}=0.84 ; n=89\right)$. The pastures were long established and composed predominantly of grasses (mainly Lolium perenne, Dactylis glomerata and Phleum pratense), but also of clover (mainly Trifolium repens) and herbs (mainly Taraxacum officinale). The pastures were fertilized once per year with $25 \mathrm{~m}^{3} / \mathrm{ha}$ of farm-produced manure (corresponding approximately to $80 \mathrm{~kg} \mathrm{~N} / \mathrm{ha}, 22 \mathrm{~kg}$ P/ha and $108 \mathrm{~kg} \mathrm{~K} / \mathrm{ha}$ ). The chemical composition of the pasture during the measurement periods is presented in Table 1. 
Table 1 Average chemical composition of concentrate $(n=2)$ and pasture $(n=14)$ samples $($ mean $\pm S D)$.

\begin{tabular}{|c|c|c|c|c|c|c|c|c|}
\hline \multirow[b]{2}{*}{ Items } & \multicolumn{4}{|c|}{ Concentrate } & \multicolumn{4}{|c|}{ Pasture } \\
\hline & Period 1 & SD & Period 2 & SD & Period 1 & SD & Period 2 & SD \\
\hline DM (g/kg of wet weight) & 884 & 1.8 & 882 & 1.0 & 174 & 30.3 & 166 & 20.1 \\
\hline \multicolumn{9}{|c|}{ Analysed nutrients and mineral composition ( $\mathrm{g} / \mathrm{kg}$ of DM) } \\
\hline $\mathrm{OM}$ & 944 & 0.3 & 944 & 0.2 & 894 & 8.6 & 892 & 6.2 \\
\hline $\mathrm{CP}$ & 115 & 1.3 & 117 & 0.3 & 174 & 23.0 & 172 & 24.5 \\
\hline Ether extract & 59 & 0.1 & 61 & 1.2 & 47 & 5.3 & 49 & 5.0 \\
\hline Starch & 502 & 0.7 & 504 & 0.2 & & & & \\
\hline ADF & 79 & 2.2 & 76 & 0.7 & 249 & 20.8 & 270 & 23.4 \\
\hline NDF & 221 & 5.7 & 228 & 3.8 & 397 & 41.8 & 427 & 48.2 \\
\hline Crude fibre & 55 & 1.6 & 52 & 0.5 & 199 & 25.4 & 217 & 23.6 \\
\hline $\mathrm{Ca}$ & 8.4 & 0.11 & 8.7 & 0.06 & 8.1 & 1.40 & 8.6 & 2.2 \\
\hline$P$ & 6.2 & 0.03 & 6.3 & 0.07 & 5.1 & 0.46 & 5.2 & 0.54 \\
\hline $\mathrm{Mg}$ & 3.2 & 0.01 & 3.2 & 0.03 & 2.1 & 0.23 & 2.2 & 0.24 \\
\hline $\mathrm{Na}$ & 1.8 & 0.03 & 1.8 & 0.00 & 0.2 & 0.05 & 0.1 & 0.05 \\
\hline K & 7.9 & 0.05 & 7.9 & 0.00 & 38 & 3.4 & 35 & 1.8 \\
\hline \multicolumn{9}{|c|}{ Calculated energy and protein supply ${ }^{1}$ per kg of DM } \\
\hline NEL (MJ) & 8.1 & 0.02 & 8.2 & 0.01 & 6.3 & 0.24 & 6.1 & 0.32 \\
\hline APDE $(\mathrm{g})$ & 79 & 2.2 & 76 & 0.7 & 104 & 5.1 & 102 & 6.3 \\
\hline \multicolumn{9}{|c|}{ Analysed $n$-alkane contents (mg/kg of DM) } \\
\hline HC32 & 0.5 & 0.50 & 0.7 & 0.03 & 5.0 & 0.66 & 5.6 & 1.60 \\
\hline HC33 & 2.6 & 0.24 & 2.5 & 0.02 & 59 & 10.0 & 65 & 15.5 \\
\hline
\end{tabular}

$\mathrm{DM}=$ dry matter; $\mathrm{OM}=$ organic matter; $\mathrm{NEL}=$ net energy for lactation; $\mathrm{APDE}=$ absorbable protein in the small intestine when rumen fermentable energy is limiting microbial protein synthesis in the rumen; $\mathrm{HC} 32=$ dotriacontane, $\mathrm{C}_{32} \mathrm{H}_{66} ; \mathrm{HC} 33=$ tritriacontane, $\mathrm{C}_{33} \mathrm{H}_{68}$.

${ }^{1}$ According to Agroscope (2013).

During an adaptation period of 21 days before both measurement periods, a step-wise provision towards the targeted amount of concentrate (UFA 275 Bio; UFA AG, Herzogenbuchsee, Switzerland), 0 or $6 \mathrm{~kg}$ (as-fed basis), took place. The pelleted concentrate was offered to six $\mathrm{HCH}-\mathrm{HNZ}$ cow pairs in two equal meals $(3 \mathrm{~kg}$ at $0600 \mathrm{~h}$ and $3 \mathrm{~kg}$ at $1700 \mathrm{~h}$ ) after milking in the free-stall barn using separate buckets for each cow. During the measurement period, all $6 \mathrm{~kg}$ of concentrate was ingested by all cows with no refusals. The other six HCH-HNZ cow pairs received no concentrate in addition to pasture. Fresh water was always available and a mineral block was available in the barn.

The ambient outdoor temperature was recorded daily by the meteorological station in Grangeneuve (MeteoSchweiz, Station Grangeneuve, Switzerland), located about $15 \mathrm{~km}$ north of the experimental pastures. During the first measurement period, the average temperature was $16^{\circ} \mathrm{C}$ (minimum 13, maximum 19) and $19^{\circ} \mathrm{C}$ (minimum 15, maximum 24) in the second measurement period.

\section{Data recording and sample collection}

Milk yield (Flo-Master Pro; DeLaval AG, Sursee, Switzerland) was recorded daily and milk composition was analysed from a pooled sample of the morning and evening milk on days 1 , 4 and 7 of each data collection period. Milk samples were preserved in tubes containing Broad Spectrum Microtabs II (Gerber Instruments AG, Effretikon, Switzerland) at $8^{\circ} \mathrm{C}$. The BW was recorded twice daily after milking and BCS was assessed before each adaptation period and before and after each data collection period according to the five-point system of Edmonson et al. (1989).

To estimate individual feed intake on pasture, the $n$-alkane double-indicator technique was used (Mayes et al., 1986). Gelatin capsules (HGK 17-60 sl; Capsula GmbH, Ratingen, Germany), containing $0.5 \mathrm{~g}$ (weighing accuracy 0.001) alkane marker $\mathrm{HC} 32$ (dotriacontane, $\mathrm{C}_{32} \mathrm{H}_{66}$; Argenta Ltd, Auckland, New Zealand) on a carrier of dried fruit pomace, were administered manually with an applicator twice per day starting 6 days before the data collection periods. During the data collection period, a daily spot sample of faeces was taken from each cow with or without stimulus between 0700 and $0800 \mathrm{~h}$. Samples were pooled by cow and collection period and stored at $-20^{\circ} \mathrm{C}$. Collection of pasture samples started and ended 1 day before the faeces sampling. Pasture sample collection was carried out as described by Graf et al. (2005). Daily samples were chopped and stored at $-20^{\circ} \mathrm{C}$ until further analysis. Samples of concentrate were taken daily and pooled per data collection period.

Grazing and rumination behaviour was recorded automatically using a jaw movement recorder with a pressure sensor (Datenlogger MSR145; MSR Electronics GmbH, Hengart, Switzerland; Nydegger et al., 2011). The jaw movement frequency and amplitude were measured for $72 \mathrm{~h}$. Data were evaluated with the software programs MSRReader (MSR 5.20.01) and MSR-Viewer (Viewer V2), as described in the MSR145 User Manual (MSR Electronics $\mathrm{GmbH}$ ). The number of grazing and rumination bouts were counted manually from the evaluated output of the software 
MSR-Viewer. A bout was defined as a sequence of a behaviour not interrupted by any other element of behaviour with a duration $>4$ min (Metz, 1975) and an inter-bout interval $>7.5$ min (Dado and Allen, 1994). Grazing bouts contain only those bouts during grazing on pasture not considering bouts when concentrate was eaten in the barn.

Physical activity, including time spent standing, lying and walking was determined using the IceTag ${ }^{\mathrm{TM}}$ pedometer (IceRobotics Ltd, Edinburgh, Scotland, UK). The pedometer was attached to the right hind leg of the cow at the metatarsus level and recorded acceleration in three dimensions at $0.1 \mathrm{~s}$ intervals for $72 \mathrm{~h}$. Using the software program IceTag-Analyser (V 4.005; IceRobotics Ltd), the data were downloaded and compiled over $60 \mathrm{~s}$ intervals. Walking was defined as $>3$ steps/min, as suggested by Thanner et al. (2014).

On days 4 and 5 of each data collection period, blood was collected at 0700 and $1400 \mathrm{~h}$ by puncture of the jugular vein, using the Vacuette ${ }^{\circledR}$ System (Greiner Bio-One GmbH, Kremsmünster, Austria). Plasma for the analysis of hormones was retrieved using Vacuette ${ }^{\circledR}$ EDTA tubes (Greiner Bio-One $\mathrm{GmbH}$ ). After sampling, these tubes were cooled in ice water until they were centrifuged at room temperature $\left(20^{\circ} \mathrm{C}\right)$ for $15 \mathrm{~min}$ at $3000 \times \mathrm{g}$. For analyses of blood metabolites and enzymes, Vacuette ${ }^{\circledR}$ serum tubes (Greiner Bio-One $\mathrm{GmbH}$ ) were stored upside down for $1 \mathrm{~h}$ at room temperature $\left(20^{\circ} \mathrm{C}\right)$ before centrifugation at $3000 \times \mathbf{g}$ for $15 \mathrm{~min}$ and then at $4000 \times \mathbf{g}$ for an additional $5 \mathrm{~min}$ (Thanner et al., 2014). The retrieved serum and plasma samples were stored at $-20^{\circ} \mathrm{C}$ until they were analysed for hormones, metabolites and enzymes.

\section{Laboratory analysis}

Milk samples were analysed by IR spectrometry (CombiFoss FT+; Foss, Hillerød, Denmark) for contents of fat, protein and lactose (International Dairy Federation, 2000; method number 141C). Urea was determined with a differential $\mathrm{pH}$ analyser (Eurochem, Ardea, Italy) before and after hydrolysis with urease (International Dairy Federation, 2004; method number 195). For milk acetone determination, acetone and an internal standard (2-butanone) were transferred via static headspace directly from the milk into the gas phase. The composition of the gas phase was determined with a flame ionization detector on a gas chromatograph (HP 5890 Series II; Agilent Technologies, Santa Clara, CA, USA).

Pasture and faeces samples were lyophilized (Christ, Delta 1-24LSC; Martin Christ Gefriertrocknungsanlagen GmbH, Osterode am Harz, Germany). Concentrate, pasture and faeces samples were milled through a $1.0-\mathrm{mm}$ screen (Brabender mill with titanium blades; Brabender, Duisburg, Germany). Subsamples were dried for $3 \mathrm{~h}$ at $105^{\circ} \mathrm{C}$ to determine DM and subsequently incinerated at $550^{\circ} \mathrm{C}$ until they reached a stable mass to assess the ash contents. Mineral residues in the ash were dissolved with nitric acid and analysed for $\mathrm{Ca}, \mathrm{Na}, \mathrm{P}, \mathrm{Mg}$ and $\mathrm{K}$ with inductively coupled plasma optical emission spectrometry (ICP-OES Optima 2000 DV; PerkinElmer, Shelton, CT, USA with system ICP-OES Optima 7300) based on European Standard: EN 155510:2008. The contents of $n$-alkanes HC32 and HC33 (tritriacontane, $\mathrm{C}_{33} \mathrm{H}_{68}$ ) were determined as described by Peiretti et al. (2006). The $\mathrm{N}$ content was determined using the Dumas method (Association of Official Analytical Chemists (AOAC), 1995) on a C/N analyser (type FP-2000; Leco Instruments, St. Joseph, MI, USA) and then multiplied by 6.25 to determine the $\mathrm{CP}$ content. The ether extract was determined using the Soxtec Avanti 2050 apparatus (Foss, Hillerød, Denmark) for extraction following the guidelines of Verband Deutscher Landwirtschaftlicher Untersuchungs- und Forschungsanstalten 5.1.1. (1993). Acid-detergent fibre (procedure 973.18; AOAC, 1995) was determined with correction for residual ash obtained after incineration at $500^{\circ} \mathrm{C}$ for $1 \mathrm{~h}$. Crude fibre was analysed in pasture and concentrate (procedure 978.10; AOAC, 1995) and NDF (Mertens, 2002) was assessed with the addition of heat-stable amylase and sodium sulphite. Starch content was determined based on the polarimetric method (method 6493; International Organization for Standardization, 2000).

Metabolite concentrations and enzyme activities were determined using the following commercial test kits: albumin (no. 11970909; Roche Diagnostics, Rotkreuz, Switzerland), alkaline phosphatase (AP, no. 12173107; Roche Diagnostics), alanine aminotransferase (ALAT, no. 63212; bioMérieux, Marcy-l'Etoile, France), aspartate aminotransferase (ASAT, no. 63212; bioMérieux), $\beta$-hydroxybutyrate (BHBA; no. RB1007; Randox Laboratories, Crumlin, UK), cholesterol (no. 61218; bioMérieux), creatinine (no. 11489291216; Roche Diagnostics), creatine kinase (CK, no. 61141; bioMérieux), $\gamma$-glutamyltransferase (GGT, no. 2016788; Roche Diagnostics), glutamate dehydrogenase (GLDH, no. 1929992; Roche Diagnostics), total protein (no. 1553836; Roche Diagnostics), urea (no. 61974, UV 250; bioMérieux), triglyceride (no. 61236; bioMérieux), nonesterified fatty acids (NEFA, no. FA 115; Randox Laboratories) and glucose (no. 1447513; Roche Diagnostics).

Plasma insulin and IGF-1 concentrations were quantified using radioimmunoassay as described by Vicari et al. (2008). 3,5,3'-triiodthyronine (T3) and thyroxin (T4) were measured by radioimmunoassay using the Coat- $\mathrm{A}-\mathrm{Count}^{\circledR}{ }^{\circledR}$ Total $\mathrm{T} 3 \mathrm{kit}$ and Coat-A-Count ${ }^{\circledR}$ Total T4 kit, respectively (Siemens Schweiz AG, Zurich, Switzerland).

\section{Calculations and statistical analyses}

Net energy for lactation (NEL) and the absorbable protein in the small intestine when rumen fermentable energy is limiting microbial protein synthesis in the rumen were calculated according to Agroscope (2013). The energy-corrected milk yield (ECM) was calculated based on a $4 \%$ fat, $3.2 \%$ protein and $4.8 \%$ lactose according to Agroscope (2013). Feed intake was calculated using the ratio of the $n$-alkanes HC32 and $\mathrm{HC} 33$ on the basis of the equation proposed by Mayes et al. (1986).

To double check the intake estimation, the pasture intake without (equation (1), DMI ${ }_{\text {conco }}$ ) and with concentrate supplementation (equation (2), $\mathrm{DMI}_{\text {conc6 }}$ ) was additionally calculated according to Baker (2004) based on the recommendations of Agroscope (2013). Changes in BW were not 
considered, as a period of 1 week is too short to estimate these accurately:

$$
\mathrm{DMI}_{\text {conc } 0}=\left(0.293 \times \mathrm{BW}^{0.75}+3.14 \times \mathrm{ECM}\right) / \text { NEL pasture } \text { or }
$$

$$
\begin{aligned}
\mathrm{DMI}_{\text {conc6 }}= & \left(0.293 \times \mathrm{BW}^{0.75}+3.14 \times \mathrm{ECM}\right. \\
& -\mathrm{NEL} \text { Conc } \times 5.3 \mathrm{~kg} \mathrm{DM}) / \mathrm{NEL} \text { pasture }
\end{aligned}
$$

where $\mathrm{BW}^{0.75}$ is the metabolic body size (kg BW to the power $0.75), E C M(\mathrm{~kg} / \mathrm{d})$ the energy-corrected milk yield, NEL Conc the NEL content of the concentrate $(\mathrm{MJ} / \mathrm{kg} \mathrm{DM})$ and NEL pasture the NEL content of the pasture (MJ/kg DM).

The statistical analyses were carried out with SYSTAT 13 (Systat Software Inc., Chicago, IL, USA). Data for milk yield and composition, rumination and grazing behaviour, physical activity, feed intake and blood traits were collected over several days and averaged per cow and measurement period. They were analysed using following linear mixed model:

$Y_{i j k l m}=\mu+\tau_{i}+\varphi_{j}+P_{k}+(\tau p)_{i k}+(\tau \varphi)_{i j}+P_{I}+K_{m}\left(P_{l}\right)+\epsilon_{i j k l m}$ where $Y_{i j k l m}$ is the response, $\mu$ the least-squares mean, $\tau_{i}$ the fixed effect of cow strain $i(i=\mathrm{HCH}, \mathrm{HNZ}), \varphi_{j}$ the fixed effect of the treatment $j(j=$ conc 0 , conc 6$), P_{k}$ the fixed effect of the period $(k=$ period 1 , period 2$),(\tau p)_{i k}$ the effect of the interaction between cow strain $i$ and period $k_{r}(\tau \varphi)_{i j}$ the effect of the interaction between cow strain $i$ and treatment $j$, $P_{\text {I }}$ the random effect of cow pair $I(1, \ldots, 12), K_{m}$ the random effect of the cow $m(1, \ldots, 24)$ and $\varepsilon_{i j k m}$ the random error. Models of this type were recommended by Tempelman (2004) with variance components as variance-covariance structure for the repeated measurements. As there were only two periods no alternative variance-covariance structure was envisaged in this study. Not normally distributed data were either logarithmically transformed to fit with normal distribution (NEFA and creatinine kinase) or analysed using $R$ ( $R$ Core Team, 2012) with permutation tests for linear models (lactose; Good, 2005). Data presented in the tables were back transformed.

Due to the high proportion of insulin 'non-detects' (i.e. values below $3 \mu \mathrm{U} / \mathrm{ml})$, descriptive statistics was performed as described by Helsel (2012) using R (R Core Team, 2012). Inferential statistics were based on rank methods (Brunner et al., 2002). The model was of F1-LD-F2 type (one betweensubject factor, two within-subject factors) and ANOVA-type test statistics (Brunner et al., 2002) were applied.

The effects were considered significant at $P \leqslant 0.05$. A value of $0.05<P<0.10$ was considered a trend.

\section{Results}

Milk yield and milk composition and feed intake

Non-supplemented cows produced less $(P<0.001)$ milk and less $(P<0.001)$ ECM compared with supplemented cows and HNZ cows had a lower $(P=0.04)$ milk yield compared with $\mathrm{HCH}$ cows, but no difference between cow strains was observed for ECM (Table 2). An interaction $(P=0.02)$ between cow strain and supplementation was reported for milk yield. The HNZ cows produced less $(P=0.04)$ milk per $\mathrm{kg}$

\begin{tabular}{|c|c|c|c|c|c|c|c|c|}
\hline \multirow[b]{2}{*}{ Items } & \multicolumn{2}{|c|}{ Conc0 } & \multicolumn{2}{|c|}{ Conc6 } & \multirow[b]{2}{*}{ SD } & \multicolumn{3}{|c|}{$P$-value } \\
\hline & $\mathrm{HCH}$ & HNZ & $\mathrm{HCH}$ & HNZ & & Treatment & Cow strain & Interaction \\
\hline \multicolumn{9}{|l|}{ Milk production performance } \\
\hline Milk yield $(\mathrm{kg} / \mathrm{d})$ & $24.9^{\mathrm{A}}$ & $23.0^{\mathrm{B}}$ & $30.0^{C}$ & $26.0^{\mathrm{D}}$ & 4.8 & $<0.001$ & 0.04 & 0.02 \\
\hline Milk yield concentrate ${ }^{1}(\mathrm{~kg} / \mathrm{kg})$ & & & $0.84^{\mathrm{a}}$ & $0.50^{b}$ & 0.30 & & 0.04 & \\
\hline $\mathrm{ECM}^{2}(\mathrm{~kg} / \mathrm{d})$ & $22.8^{\mathrm{A}}$ & $22.6^{\mathrm{A}}$ & $25.3^{\mathrm{B}}$ & $23.9^{\mathrm{B}}$ & 3.8 & $<0.001$ & 0.41 & 0.16 \\
\hline Fat $(\%)$ & $3.7^{\mathrm{A}}$ & $4.0^{\mathrm{A}}$ & $3.0^{B}$ & $3.4^{\mathrm{B}}$ & 0.6 & $<0.001$ & 0.08 & 0.50 \\
\hline Protein (\%) & $3.2^{\mathrm{A}}$ & $3.4^{\mathrm{B}}$ & $3.2^{\mathrm{A}}$ & $3.5^{\mathrm{B}}$ & 0.3 & 0.13 & $<0.01$ & 0.96 \\
\hline Lactose $^{3}(\%)$ & 4.4 & 4.4 & 4.5 & 4.5 & 0.3 & 0.39 & 0.50 & 0.74 \\
\hline Acetone (mg/l) & $2.87^{\mathrm{A}}$ & $2.53^{\mathrm{A}}$ & $1.52^{\mathrm{B}}$ & $1.36^{\mathrm{B}}$ & 0.79 & $<0.001$ & 0.27 & 0.68 \\
\hline Urea $(\mathrm{mg} / \mathrm{l})$ & $239^{\mathrm{A}}$ & $226^{\mathrm{A}}$ & $178^{\mathrm{B}}$ & $176^{\mathrm{B}}$ & 30.0 & $<0.001$ & 0.49 & 0.34 \\
\hline \multicolumn{9}{|l|}{ Feed intake } \\
\hline Pasture DMI (kg DM/day) & $12.5^{\mathrm{A}}$ & $11.7^{\mathrm{A}}$ & $9.7^{\mathrm{B}}$ & $9.0^{\mathrm{B}}$ & 1.7 & $<0.001$ & 0.09 & 0.83 \\
\hline Total DMI (kg DM/day) & $12.5^{\mathrm{A}}$ & $11.7^{\mathrm{A}}$ & $15.0^{B}$ & $14.3^{\mathrm{B}}$ & 1.7 & $<0.001$ & 0.09 & 0.83 \\
\hline Calculated pasture $\mathrm{DMI}^{4}$ (kg DM/day) & $17.3^{\mathrm{A}}$ & $16.9^{\mathrm{A}}$ & $12.0^{\mathrm{B}}$ & $11.0^{\mathrm{B}}$ & 2.4 & $<0.001$ & 0.23 & 0.17 \\
\hline Calculated total DMI ${ }^{5}$ (kg DM/day) & 17.3 & 16.9 & 17.3 & 16.3 & 2.4 & 0.23 & 0.23 & 0.17 \\
\hline
\end{tabular}
concentrate than $\mathrm{HCH}$ cows.

Table 2 Effect of concentrate supplementation and cow strain and their interactions on milk production performance and feed intake

Conc 0 = cows without concentrate supplementation; Conc $6=$ cows with concentrate supplementation; HCH = Swiss Holstein-Friesian; HNZ = New Zealand Holstein-Friesian; $\mathrm{DMI}=$ dry matter intake; $\mathrm{DM}=$ dry matter.

$A, B, C, D$ Means with different superscript letters within the same row differ $(P<0.01)$.

a,b Means with different superscript letters within the same row differ $(P<0.05)$.

${ }^{1}$ Milk yield per ingested concentrate $(\mathrm{kg} / \mathrm{kg})$.

${ }^{2} \mathrm{ECM}=$ energy-corrected milk yield (Agroscope, 2013).

${ }^{3} \log _{10}$ transformed for statistical analyses.

${ }^{4}$ According to Agroscope (2013) and Baker (2004) without BW changes and activity.

${ }^{5}$ According to Agroscope (2013) and Baker (2004) without BW changes and activity plus $5.3 \mathrm{~kg} \mathrm{DM}$ of concentrate. 
Milk fat content was greater $(P<0.001)$ for nonsupplemented cows, but milk protein content was not influenced by supplementation. The HNZ cows had a greater $(P<0.01)$ protein content and in tendency a greater milk fat content than $\mathrm{HCH}$ cows. No differences were recorded for the lactose content relative to concentrate supplementation or cow strain. The milk contents of acetone and urea of non-supplemented cows were greater $(P<0.001)$ compared with supplemented cows, but no differences were observed between cow strains.

For non-supplemented cows, pasture dry matter intake (DMI) estimated with $n$-alkanes was greater $(P<0.001)$ than pasture DMI for supplemented cows, but total DMI estimated with alkanes was lower $(P<0.001)$ for non-supplemented cows than for supplemented cows. New Zealand Holstein cows tended to have lower pasture DMI and total DMI compared with $\mathrm{HCH}$ cows. Calculated pasture DMI based on the requirements was greater $(P<0.001)$ for nonsupplemented cows, but supplementation had no effect on calculated total DMI and no differences between cow strains were observed for calculated pasture and total DMI.

\section{Grazing and rumination behaviour and physical activity} Grazing time, mastication $(n)$ and mastication rate $(n / \mathrm{min})$ were greater $(P<0.001)$ for non-supplemented cows compared with supplemented cows and no differences occurred between cow strains (Table 3). The number of grazing bouts was not affected by supplementation, but HNZ cows tended to have greater numbers compared with $\mathrm{HCH}$ cows. Non-supplemented cows had a greater $(P<0.001)$ duration of eating bouts compared with supplemented cows, but no difference between cow strains was reported.

Concentrate supplementation had no effect on traits describing rumination behaviour, but HNZ cows spent more $(P<0.005)$ time ruminating, had a greater $(P<0.005)$ number of mastication and a greater $(P<0.005)$ number of boli compared with $\mathrm{HCH}$ cows. No difference between cow strains was observed for mastication rate, mastication per boli and rumination bouts. New Zealand Holstein cows tended to have longer duration of rumination bouts compared with $\mathrm{HCH}$ cows.

Non-supplemented cows spent less $(P<0.001)$ time lying down, but stood, moved and walked more $(P<0.001)$. There was a trend recorded for HNZ to stand and move less and lie down more, but no difference was observed for walking. No significant interaction of concentrate supplementation and cow strain was observed.

\section{Blood traits}

Non-supplemented cows had lower $(P<0.01)$ serum glucose concentration but greater $(P<0.001)$ concentration of serum BHBA, NEFA and urea compared with supplemented cows (Table 4). Concentration of total protein in serum indicated a tendency to be affected by supplementation, with a greater concentration for non-supplemented cows. Concentrate supplementation had no effect on concentration of serum albumin, triglycerides, cholesterol and creatinine. The activity of creatinine kinase, GLDH and GGT was not affected by

Table 3 Effect of concentrate supplementation and cow strain and their interaction on grazing and rumination behaviour and physical activity over $24 h$

\begin{tabular}{|c|c|c|c|c|c|c|c|c|}
\hline \multirow[b]{2}{*}{ Items } & \multicolumn{2}{|c|}{ Conc0 } & \multicolumn{2}{|c|}{ Conc6 } & \multirow[b]{2}{*}{ SD } & \multicolumn{3}{|c|}{$P$-value } \\
\hline & $\mathrm{HCH}$ & HNZ & $\mathrm{HCH}$ & HNZ & & Treatment & Cow strain & Interaction \\
\hline \multicolumn{9}{|l|}{ Grazing behaviour over $24 \mathrm{~h}$} \\
\hline Time (min) & $547.1^{\mathrm{A}}$ & $568.7^{\mathrm{A}}$ & $442.9^{B}$ & $453.0^{B}$ & 43.9 & $<0.001$ & 0.20 & 0.63 \\
\hline Mastications $(n)$ & $41232^{A}$ & $42102^{A}$ & $32070^{B}$ & $32865^{\mathrm{B}}$ & 3859 & $<0.001$ & 0.51 & 0.97 \\
\hline Mastication rate $(\mathrm{n} / \mathrm{min})$ & $75.3^{\mathrm{A}}$ & $74.0^{\mathrm{A}}$ & $72.5^{\mathrm{B}}$ & $72.7^{\mathrm{B}}$ & 4.0 & $<0.001$ & 0.73 & 0.06 \\
\hline Grazing bouts $(n)$ & 5.2 & 5.7 & 5.5 & 6.0 & 0.94 & 0.24 & 0.10 & 0.81 \\
\hline Duration of grazing bouts (min) & $110^{\mathrm{A}}$ & $104^{\mathrm{A}}$ & $81^{\mathrm{B}}$ & $78^{\mathrm{B}}$ & 18.8 & $<0.001$ & 0.42 & 0.77 \\
\hline \multicolumn{9}{|l|}{ Rumination behaviour over $24 \mathrm{~h}$} \\
\hline Time (min) & $381^{a}$ & $405^{b}$ & $398^{\mathrm{a}}$ & $423^{b}$ & 40.8 & 0.15 & $<0.05$ & 0.99 \\
\hline Mastications $(n)$ & $27661^{a}$ & $29796^{b}$ & $28888^{a}$ & $31204^{b}$ & 3932 & 0.20 & 0.04 & 0.93 \\
\hline Mastication rate ( $\mathrm{n} / \mathrm{min})$ & 72.4 & 73.4 & 72.7 & 73.7 & 5.3 & 0.47 & 0.48 & 0.92 \\
\hline Rumination boli $(n)$ & $522^{\mathrm{a}}$ & $578^{b}$ & $551^{\mathrm{a}}$ & $597^{b}$ & 69.2 & 0.22 & 0.04 & 0.79 \\
\hline Mastications boli ( $n /$ boli) & 51.5 & 53.8 & 54.9 & 53.4 & 5.0 & 0.26 & 0.83 & 0.17 \\
\hline Rumination bouts $(n)$ & 13.1 & 12.2 & 13.1 & 12.7 & 1.58 & 0.56 & 0.14 & 0.48 \\
\hline Duration of rumination bouts (min) & 29 & 35 & 31 & 34 & 7.0 & 0.95 & 0.08 & 0.40 \\
\hline \multicolumn{9}{|l|}{ Activity over $24 \mathrm{~h}$} \\
\hline Lying (min) & $492^{A}$ & $541^{\mathrm{A}}$ & $564^{\mathrm{B}}$ & $590^{\mathrm{B}}$ & 62 & $<0.001$ & 0.05 & 0.41 \\
\hline Standing + moving (min) & $949^{A}$ & $900^{A}$ & $877^{\mathrm{B}}$ & $851^{\mathrm{B}}$ & 62 & $<0.001$ & 0.05 & 0.41 \\
\hline Walking (min) & $421^{\mathrm{A}}$ & $419^{A}$ & $373^{\mathrm{B}}$ & $362^{\mathrm{B}}$ & 59 & $<0.001$ & 0.75 & 0.73 \\
\hline
\end{tabular}

Conc 0 = cows without concentrate supplementation; Conc6 = cows with concentrate supplementation; HCH = Swiss Holstein-Friesian; HNZ = New Zealand Holstein-Friesian.

${ }_{A}^{A}, \mathrm{~B}$ Means with different superscript letters within the same row differ $(P<0.01)$.

${ }^{a, b}$ Means with different superscript letters within the same row differ $(P<0.05)$. 
Response of two cow strains to supplementation

Table 4 Effect of concentrate supplementation and cow strain and their interaction on blood metabolites, enzymes and hormones

\begin{tabular}{|c|c|c|c|c|c|c|c|c|}
\hline \multirow[b]{2}{*}{ Items } & \multicolumn{2}{|c|}{ Conc0 } & \multicolumn{2}{|c|}{ Conc6 } & \multirow[b]{2}{*}{ SD } & \multicolumn{3}{|c|}{$P$-value } \\
\hline & $\mathrm{HCH}$ & HNZ & $\mathrm{HCH}$ & HNZ & & Treatment & Cow strain & Interaction \\
\hline Glucose (mmol/l) & $3.15^{\mathrm{A}}$ & $3.31^{\mathrm{B}}$ & $3.25^{C}$ & $3.46^{\mathrm{D}}$ & 0.18 & $<0.01$ & $<0.01$ & 0.54 \\
\hline $\mathrm{BHBA}(\mathrm{mmol} / \mathrm{l})$ & $0.91^{\mathrm{A}}$ & $0.82^{\mathrm{A}}$ & $0.68^{\mathrm{B}}$ & $0.69^{B}$ & 0.17 & $<0.001$ & 0.45 & 0.30 \\
\hline $\mathrm{NEFA}^{1}(\mathrm{mmol} / \mathrm{l})$ & $0.12^{\mathrm{A}}$ & $0.14^{\mathrm{A}}$ & $0.08^{\mathrm{B}}$ & $0.09^{B}$ & 0.05 & $<0.001$ & 0.48 & 0.62 \\
\hline Urea (mmol/l) & $4.86^{\mathrm{A}}$ & $4.77^{\mathrm{A}}$ & $3.68^{\mathrm{B}}$ & $3.73^{\mathrm{B}}$ & 0.90 & $<0.001$ & 0.95 & 0.72 \\
\hline Total protein (g/l) & 73.6 & 72.9 & 72.0 & 72.2 & 4.20 & 0.10 & 0.88 & 0.48 \\
\hline Albumin (g/l) & 38.8 & 39.2 & 38.0 & 38.9 & 2.40 & 0.27 & 0.32 & 0.56 \\
\hline Triglycerides (mmol/l) & 0.30 & 0.33 & 0.30 & 0.31 & 0.08 & 0.44 & 0.28 & 0.74 \\
\hline Cholesterol (mmol/l) & 6.28 & 6.26 & 6.08 & 6.43 & 1.10 & 0.94 & 0.70 & 0.24 \\
\hline Creatinine $(\mu \mathrm{mol} / \mathrm{l})$ & 73.8 & 70.7 & 76.2 & 69.5 & 8.2 & 0.66 & 0.07 & 0.19 \\
\hline Creatine kinase $^{1}(\mathrm{U} / \mathrm{l})$ & 153 & 191 & 146 & 176 & 55.2 & 0.26 & 0.13 & 0.79 \\
\hline GLDH (U/I) & 14.4 & 16.1 & 13.1 & 16.7 & 4.11 & 0.71 & 0.08 & 0.34 \\
\hline GGT (U/l) & 22.8 & 24.7 & 22.8 & 24.8 & 4.60 & 0.90 & 0.20 & 0.97 \\
\hline ASAT (U/l) & $71.2^{\mathrm{a}}$ & $74.8^{\mathrm{a}}$ & $67.6^{\mathrm{b}}$ & $72.8^{\mathrm{b}}$ & 7.38 & 0.04 & 0.15 & 0.52 \\
\hline AP (U/l) & $39.1^{\mathrm{A}}$ & $50.6^{\mathrm{A}}$ & $48.9^{B}$ & $61.2^{\mathrm{B}}$ & 21.20 & $<0.001$ & 0.17 & 0.86 \\
\hline ALAT (U/l) & $29.2^{\mathrm{A}}$ & $31.7^{\mathrm{B}}$ & $26.6^{C}$ & $29.5^{\mathrm{D}}$ & 2.60 & $<0.001$ & $<0.01$ & 0.65 \\
\hline IGF-1 (ng/ml) & $91^{\mathrm{a}}$ & $109^{b}$ & $113^{c}$ & $141^{d}$ & 31.5 & $<0.001$ & $<0.05$ & 0.25 \\
\hline T3 (nmol/l) & $2.28^{\mathrm{A}}$ & $2.36^{A}$ & $2.45^{\mathrm{B}}$ & $2.60^{B}$ & 0.31 & $<0.001$ & 0.18 & 0.34 \\
\hline $\mathrm{T} 4$ (nmol/l) & $49.0^{\mathrm{a}}$ & $48.7^{\mathrm{a}}$ & $51.6^{\mathrm{b}}$ & $53.5^{\mathrm{b}}$ & 12.01 & 0.04 & 0.84 & 0.51 \\
\hline Insulin ( $\mu \mathrm{U} / \mathrm{ml})$ & $4.31^{a}$ & $3.81^{\mathrm{a}}$ & $5.82^{b}$ & $5.92^{b}$ & 3.54 & 0.03 & 0.94 & 0.58 \\
\hline
\end{tabular}

Conc0 = cows without concentrate supplementation; Conc6 = cows with concentrate supplementation; HCH = Swiss Holstein-Friesian; HNZ = New Zealand HolsteinFriesian; NEFA = non-esterified fatty acids; GLDH = glutamate dehydrogenase; GGT = $\gamma$-glutamyltransferase; ASAT = aspartate aminotransferase; AP = alkaline phosphatase; ALAT = alanine aminotransferase; $\mathrm{T} 3=3,5,3$ '-triiodthyronine; $\mathrm{T} 4=$ thyroxin.

$A, B, C, D$ Means with different superscript letters within the same row differ $(P<0.01)$.

$a, b, c, d$ Means with different superscript letters within the same row differ $(P<0.05)$.

${ }^{1} \log _{10}$ transformed for statistical analyses.

concentrate supplementation, but activity of ASAT and ALAT were greater $(P=0.04)$ and activity of AP was lower $(P<0.001)$ for non-supplemented cows.

Concentrate supplementation had an impact on the plasma concentration of the hormones T3 and T4 with lower $(P<0.001$ and $P=0.04$, respectively) concentrations for non-supplemented cows. Non-supplemented cows had a lower $(P=0.03)$ plasma insulin and lower $(P<0.001)$ IGF-1 concentration compared with supplemented cows.

Differences between cow strains were recorded for serum glucose concentration with lower $(P<0.01)$ concentration for $\mathrm{HCH}$ cows. Furthermore, $\mathrm{HCH}$ cows had a lower $(P<0.01)$ activity of ALAT and a lower $(P<0.05)$ concentration of IGF-1. For other blood traits no differences between cow strains were recorded. No interactions of concentrate supplementation and cow strain for blood traits were observed.

\section{Discussion}

Many studies investigated the effects of concentrate supplementation on intake, milk production, body condition, grazing behaviour and digestion under non-organic farming conditions, reviewed by Bargo et al. (2003) for grazing dairy cows. Studies under organic farming conditions focussed more on the effects on health and fertility on farm level without considering the basic effects of concentrate supplementation (Sehested et al., 2003; Ertl et al., 2014; Ivemeyer et al., 2014). According to the knowledge of the authors no other study investigated the effect of concentrate supplementation on the eating and rumination behaviour, intake, metabolic states and milk production of grazing dairy cows under organic farming conditions. Changes in organic herbage quality, as found by Spann et al. (2007), might be partly due to the principles of organic agriculture (International Federation of Organic Agriculture Movements, 2015), for instance, prohibition of synthesized fertilizers and pesticides, as well as with the general promotion of natural, multispecies pastures. As the nutritive value and the fibre content could influence the outcome of concentrate supplementation on grazing dairy cows, studies like ours are needed to close the gap. Finally, only few studies investigated the grazing and rumination behaviour, especially bites per rumination bolus, when investigating the effects of concentrate supplementation of grazing dairy cows.

\section{Effect of concentrate and consequences for pasture-based organic farming}

In accordance with Bargo et al. (2003), milk yield and ECM increased for supplemented cows, but to a smaller extent. The observed interaction between concentrate supplementation and cow strain for milk production indicate the different genetic potential for milk production between the two cow strains (Bargo et al., 2002; Horan et al., 2005). Supplemented $\mathrm{HCH}$ cows responded to the extra energy 
supply with greater milk yield compared with HNZ cows, but ECM was similar.

The reduced milk fat content of supplemented cows is in agreement with other studies, when cows received $>4 \mathrm{~kg} /$ day of concentrate (Bargo et al., 2002; Horan et al., 2005). Furthermore, the increased activity of plasma AP in supplemented cows may indicate acidotic stress. To ensure rumen health, rumination is a key factor as it influences salivation and therefore rumen buffering. In the current study, concentrate supplementation had no effect on rumination behaviour. It would have been expected that with increasing pasture DMl, time ruminating, rumination mastication and number of boli increased (Beauchemin and Rode, 1997; McCarthy et al., 2007b). The absence of differences in rumination mastication per bolus and number of boli may indicate a similar ease of bolus formation and swallowing for both supplemented and non-supplemented cows, although higher grain diets reflect a greater ease of bolus formation (Beauchemin and Rode, 1997). Rumination mastication per bolus was in the normal range. This poses the question of whether characteristics of rumination behaviour are a suitable indicator of sufficient fibre supply and therefore rumen health, at least in grazing dairy cows.

In agreement with other studies, supplementation caused a substantial reduction in grazing time (Bargo et al., 2002; McCarthy et al., 2007b) and therefore reduced grazing mastication and grazing mastication rate. This implies the lower motivation of supplemented cows to graze and is supported by the reduced duration of grazing bouts for supplemented cows, as grazing time or grazing time coupled with intake rate (bite rate and size) at the same BW might be indicators for the feeding drive (McCarthy et al., 2007b; Prendiville et al., 2010).

In accordance with other studies, pasture DMI decreased with concentrate supplementation, which is expressed as substitution rate (Bargo et al., 2002; McCarthy et al., 2007b). Both calculated and estimated (using $n$-alkanes) pasture DMI were lowered by concentrate supplementation. In contrast, total DMI of supplemented cows was greater or at least the same as the total DMI of non-supplemented cows. However, with reduced pasture DMI for supplemented cows, cheap pasture is substituted by expensive concentrate which is an economic aspect for the farmer. Furthermore, importing ingredients of a commercial organic concentrate mix disturbs the holistic approach of organic guidelines. In the current study, the energy:protein ratio may be balanced as concentrations of urea in blood and milk were in the normal range, with elevated values for nonsupplemented cows. In organic farming, the risk of excessive protein intake from pasture might be reduced as the $\mathrm{CP}$ content of organic pasture is lower compared with conventional pasture (Spann et al., 2007).

Current results indicate a strong relationship between physical activity and grazing behaviour. As supplemented cows spent less time grazing, they spent less time standing and walking. In a pasture-based feeding system, where energy might be the first limiting nutrient, physical activity on pasture is an important factor to be considered. Grazing cows have a greater energy expenditure compared with cows fed indoors, as grazing cows take more steps, spend less time lying down and spend more time eating (Kaufmann et al., 2011). According to the Commonwealth Scientific and Industrial Research Organisation (2007), energy requirements for maintenance may increase in the range of $10 \%$ to $50 \%$ depending on grazing conditions, digestibility of pasture, distance walked, weather, topography and interactions between these factors. Thus, supplemented cows on pasture did not only ingest more energy, but also presented improved energy balance due to energy savings in relation to shorter grazing and physical activities.

A lack of energy in early lactation can cause metabolic problems such as ketosis. In the current study, the increased acetone concentration in milk for non-supplemented cows indicates a small risk of ketosis. This is confirmed by an increased concentration of BHBA and NEFA and decreased glucose and insulin concentration for non-supplemented cows. The increased blood glucose, insulin and IGF-1 concentrations for supplemented cows indicate an increased energy status, whereas increased concentration of blood NEFA and BHBA for non-supplemented cows suggest a lower energy status (Reist et al., 2002). As the concentration of T3 and T4 decreases with stronger negative energy balance (NEB) (Huszenicza et al., 2002), non-supplemented cows in current study had lower concentration of T3 and T4 and therefore had a lower energy status compared with supplemented cows. Furthermore, the increased activity of ALAT of non-supplemented cows indicates an increased use of amino acids as an energy source or for gluconeogenesis (Garber et al., 1976). The greater activity of ASAT of non-supplemented cows alone should be interpreted with caution. As the activities of GLDH, GGT and CK were not increased for non-supplemented cows, the occurrence of fatty liver syndrome in cows of this study is unlikely.

Results of blood traits indicate that non-supplemented cows were not in strong NEB. Supplementation may not be necessary to balance an organic pasture-based diet, at least for cows in mid-lactation and therefore more severe, but more flexible restrictions over the whole lactation for supplementation are favourable.

Finally, it can be stated that the effects of concentrate supplementation on milk yield, milk composition, grazing behaviour and intake are similar in the present organic study compared with the cited conventional studies.

\section{Effect of cow strain and consequences for pasture-based organic farming}

Another aspect of organic farming is the choice of the breed or strain. This implies the selection of cows adapted to lowinput pasture or forage-based feeding system for organic dairy production. In the present study, the milk yield responses obtained for $\mathrm{HNZ}$ and $\mathrm{HCH}$ cows were similar to the results of Horan et al. (2005). Swiss Holstein cows reached almost the overall milk yield response of $1 \mathrm{~kg}$ milk/ $1 \mathrm{~kg}$ concentrate as published by Bargo et al. (2003), but not in ECM terms. The lower genetic potential for milk 
production of HNZ cows might be the reason for their lower response of $0.5 \mathrm{~kg}$ milk/kg of ingested concentrate, as pasture allowance and mass was the same for both strains. Cows with high genetic potential for milk yield have a greater milk yield response to concentrate supplementation (McCarthy et al., 2007b). This phenomenon might be attributed to greater nutrient partition to milk production in high genetic merit cows compared with lower genetic merit cows (Dillon et al., 2006). This is supported by the results of IGF-1, with greater plasma concentration in HNZ cows. Greater IGF-1 plasma concentration indicates the coupling of the somatotropic axis, as the liver responds to increased growth hormone concentration and therefore nutrient partitioning favours the build up of body tissue instead of milk production (Lucy et al., 2009).

In contrast to results for milk yield, no difference for ECM between cow strains was observed which can be explained by the greater milk protein and in trend greater milk fat content of the HNZ cows. Similarity of ECM yields of the two cow strains indicate a similar efficiency in milk production for $\mathrm{HCH}$ and HNZ cows in the present study.

New Zealand Holstein cows ruminated longer than $\mathrm{HCH}$ cows, which was also observed in previous studies (Schori and Münger, 2014; Thanner et al., 2014). In line with this result HNZ cows tended to spend more time lying down compared with $\mathrm{HCH}$ cows as rumination of cows on pasture is associated with lying down (Kilgour, 2012). Furthermore, HNZ cows had a greater number of boli and greater rumination mastication per day compared with $\mathrm{HCH}$ cows. Anatomical differences of the muzzle and incisor breadth might explain this (Rook, 2000). Prendiville et al. (2010) observed smaller bolus size for the smaller Jersey cows compared with HF cows, indicating that anatomical differences influence the pattern of bolus movement during rumination. Although greater chewing activity during grazing and rumination is associated with a greater salivary secretion and therefore a better fibre digestibility (Domingue et al., 1991), HNZ cows could not benefit from longer rumination time in terms of milk production.

The trend for lower pasture DMI by HNZ cows and the lack of clear differences in grazing behaviour do not confirm a greater feeding drive for HNZ cows (McCarthy et al., 2007b). It could have been expected that $\mathrm{HCH}$ cows had a greater pasture DMI, as DMI and BW are positively correlated (Kertz et al., 1991), as BW is usually positively linked to rumen size and therefore intake capacity. Similar pasture DMI indicates a greater DMI per kg BW for $\mathrm{HNZ}$ cows compared with $\mathrm{HCH}$ cows. Furthermore, HNZ cows spent longer time ruminating. Digestion rate and discharging of the rumen might be increased as rumination determines digestion rate and therefore controls voluntary intake (Bae et al., 1983; Gregorini et al., 2012).

The substitution rate of pasture DMI is linked to milk response with a lower substitution rate for cows with high genetic potential for milk yield (Bargo et al., 2003). However, in the current study, substitution rate, estimated using $n$ alkane, was the same for both cow strains $(0.5 \mathrm{~kg} / \mathrm{kg})$. The results are in accordance with missing differences for ECM.
Because no differences were observed between cow strains in blood concentration of insulin, NEFA and BHBA, which are indicators of the energy balance of dairy cows (Reist et al., 2002), no difference between cow strains are obvious in energy status. However, a trend for an increased concentration of creatinine in blood plasma for $\mathrm{HCH}$ cows may indicate a higher skeletal muscle breakdown and the mobilization of more protein as an energy source or for gluconeogenesis. In accordance with results from McCarthy et al. (2007a), HNZ cows had greater serum glucose concentration compared with $\mathrm{HCH}$ cows. The greater glucose concentration for HNZ cows during breeding season (60 to 150 DIM) represents an important source for energy for the ovary, as Forshell et al. (1991) reported an effect of glucose concentration on conception rates. Therefore greater glucose concentration might indicate greater conception rates which is in line with the differences in conception rates reported between HCH cows and HNZ cows (Piccand et al., 2013).

The increase in ALAT activity is in accordance with the results of Thanner et al. (2014), which might suggest the elevated use of amino acids of the HNZ cows for purposes other than protein synthesis.

Minor differences between cow strains in the current study indicate that both cow strains are equally suited for an organic, pasture-based feeding system. However, the current study was a short-term experiment performed with a small number of animals after the peak of lactation without considering BCS losses, fertility and health traits during a whole lactation, which are important aspects for continuous and successful organic farming. Just choosing a different cow breed or cow strain may not ensure a wellworking, low-input organic system. Careful selection of cows that have a high grazing drive ensures the efficient use of pasture for milk production, whereas cows that can deal with lower energy intake at the beginning of lactation and show properties of good health and fertility may be well-suited organic cows.

\section{Acknowledgements}

This study was funded by the AGFF (Zürich, Switzerland) research fund and the Fondation Sur-la-Croix (Basel, Switzerland). The authors gratefully acknowledge the help afforded to them by the staff of the farm 'Ferme École de Sorens' and C. E. Pardo for sampling and assistance with the cows during experiment. The authors thank the staff of Agroscope in Posieux and Vetsuisse Faculty of Berne for assistance in the laboratory analyses and W. Luginbühl for statistical advice.

\section{References}

Agroscope 2013. Fütterungsempfehlungen und Nährwerttabellen für Wiederkäuer (Grünes Buch). Forschungsanstalt Agroscope Liebefeld-Posieux ALP, Posieux, Switzerland.

Association of Official Analytical Chemists (AOAC) 1995. Official methods of analysis, 16th edition. AOAC, Arlington, VA, USA.

Bae DH, Welch JG and Gilman BE 1983. Mastication and rumination in relation to body size of cattle. Journal of Dairy Science 66, 2137-2141. 
Baker RD 2004. Estimating herbage intake from animal performance. In Herbage intake handbook (ed. PD Penning), pp. 95-120. The British Grassland Society, Reading, UK

Bargo F, Muller LD, Delahoy JE and Cassidy TW 2002. Milk response to concentrate supplementation of high producing dairy cows grazing at two pasture allowances. Journal of Dairy Science 85, 1777-1792.

Bargo F, Muller LD, Kolver ES and Delahoy JE 2003. Invited review: production and digestion of supplemented dairy cows on pasture. Journal of Dairy Science 86, 1-42.

Beauchemin KA and Rode LM 1997. Minimum versus optimum concentrations of fiber in dairy cow diets based on barley silage and concentrates of barley or corn. Journal of Dairy Science 80, 1629-1639.

Bundesamt für Landwirtschaft 2015. Auswertung der Daten über die Milchproduktion. Eidgenössisches Department für Wirtschaft, Bildung und Forschung WBF, BLW, Bern, Switzerland.

Brunner $E$, Domhof $S$ and Langer $F$ 2002. Nonparametric analysis of longitudinal data in factorial experiments. Wiley, New York, NY, USA.

Commonwealth Scientific and Industrial Research Organisation 2007. Energy. In Nutrient requirements of domesticated ruminants (ed. Freer $\mathrm{M}$, Dove $\mathrm{H}$ and Nolan JV), pp. 1-69. Commonwealth Scientific and Industrial Research Organisation, Collingwood, Australia.

Dado RG and Allen MS 1994. Variation in and relationships among feeding, chewing, and drinking variables for lactating dairy cows. Journal of Dairy Science 77, 132-144.

Dillon P, Berry DP, Evans RD, Buckley F and Horan B 2006. Consequences of genetic selection for increased milk production in European seasonal pasture based systems of milk production. Livestock Science 99, 141-158.

Domingue BM, Dellow DW and Barry TN 1991. The efficiency of chewing during eating and ruminating in goats and sheep. British Journal of Nutrition 65, 355-363.

Edmonson AJ, Lean IJ, Weaver LD, Farver T and Webster G 1989. A body condition scoring chart for Holstein dairy cows. Journal of Dairy Science 72, 68-78.

Ertl P, Knaus W and Steinwidder A 2014. Comparison of zero concentrate supplementation with different quantities of concentrates in terms of production, animal health, and profitability of organic dairy farms in Austria. Organic Agriculture 4, 233-242.

Forshell KP, Andersson L and Pehrson B 1991. The relationships between the fertility of dairy cows and clinical and biochemical measurements, with special reference to plasma glucose and milk acetone. Journal of Veterinary Medicine Series A 38, 608-616.

Garber AJ, Karl IE and Kipnis DM 1976. Alanine and glutamine synthesis and release from skeletal muscle. II. The precursor role of amino acids in alanine and glutamine synthesis. Journal of Biological Chemistry 251, 836-843.

Good P 2005. Permutation, parametric and bootstrap tests of hypotheses, 3rd edition. Springer, Huntington Beach, CA, USA.

Graf CM, Kreuzer M and Dohme F 2005. Effects of supplemental hay and corn silage versus full-time grazing on ruminal $\mathrm{pH}$ and chewing activity of dairy cows. Journal of Dairy Science 88, 711-725.

Gregorini P, DelaRue B, McLeod K, Clark CEF, Glassey CB and Jago J 2012. Rumination behavior of grazing dairy cows in response to restricted time at pasture. Livestock Science 146, 95-98.

Helsel DR 2012. Statistics for censored environmental data using Minitab and R, 2nd edition. Wiley, Hoboken, NJ, USA.

Horan B, Dillon P, Faverdin P, Delaby L, Buckley F and Rath M 2005. The interaction of strain of Holstein-Friesian cows and pasture-based feed systems on milk yield, body weight, and body condition score. Journal of Dairy Science 88, 1231-1243.

Huszenicza G, Kulcsar M and Rudas P 2002. Clinical endocrinology of thyroid gland function in ruminants. Veterinární Medicína 47, 199-210.

International Dairy Federation 2000. Determination of milk fat, protein and lactose content. Guide for the operation of mid-infra-red instruments. IDF Standard method No. 141C, Brussels, Belgium.

International Dairy Federation 2004. Milk. Determination of urea content. Enzymatic method using difference in pH. IDF Standard method No. 195, Brussels, Belgium.

International Federation of Organic Agriculture Movements 2015. Principles of organic agriculture. Retrieved on 20 August 2015 from http://www.ifoam.org/ en/organic-landmarks/principles-organic-agriculture.
International Organization for Standardization 2000. Animal feeding stuffs - determination of starch content - polarimetric method. ISO, Geneva, Switzerland.

Ivemeyer S, Walkenhorst M, Holinger M, Maeschli A, Klocke P, Spengler Neff A, Staehli P, Krieger M and Notz C 2014. Changes in herd health, fertility and production under roughage based feeding conditions with reduced concentrate input in Swiss organic dairy herds. Livestock Science 168, 159-167.

Kaufmann LD, Münger $A$, Rérat $M$, Junghans $P$, Görs $S$, Metges $C C$ and Dohme-Meier F 2011. Energy expenditure of grazing cows and cows fed grass indoors as determined by the ${ }^{13} \mathrm{C}$ bicarbonate dilution technique using an automatic blood sampling system. Journal of Dairy Science 94, 1989-2000.

Kertz AF, Reutzel LF and Thomson GM 1991. Dry matter intake from parturition to midlactation. Journal of Dairy Science 74, 2290-2295.

Kilgour RJ 2012. In pursuit of normal: a review of the behaviour of cattle at pasture. Applied Animal Behaviour Science 138, 1-11.

Kolver ES and Muller LD 1998. Performance and nutrient intake of high producing Holstein cows consuming pasture or a total mixed ration. Journal of Dairy Science 81, 1403-1411.

Lucy MC, Verkerk GA, Whyte BE, Macdonald KA, Burton L, Cursons RT, Roche JR and Holmes CW 2009. Somatotropic axis components and nutrient partitioning in genetically diverse dairy cows managed under different feed allowances in a pasture system. Journal of Dairy Science 92, 526-539.

Macdonald KA, Verkerk GA, Thorrold BS, Pryce JE, Penno JW, McNaughton LR, Burton LJ, Lancaster JAS, Williamson JH and Holmes CW 2008. A comparison of three strains of Holstein-Friesian grazed on pasture and managed under different feed allowances. Journal of Dairy Science 91, 1693-1707.

Mayes RW, Lamb CS and Colgrove PM 1986. The use of dosed and herbage $\mathrm{n}$-alkanes as markers for the determination of herbage intake. The Journal of Agricultural Science 107, 161-170.

McCarthy S, Berry DP, Dillon P, Rath M and Horan B 2007a. Effect of strain of Holstein-Friesian and feed system on calving performance, blood parameters and overall survival. Livestock Science 111, 218-229.

McCarthy S, Horan B, Rath M, Linnane M, O'Connor P and Dillon P 2007b. The influence of strain of Holstein-Friesian dairy cow and pasture-based feeding system on grazing behaviour, intake and milk production. Grass and Forage Science 62, 13-26.

Mertens DR 2002. Gravimetric determination of amylase-treated neutral detergent fiber in feeds with refluxing in beakers or crucibles: collaborative study. Journal of AOAC International 85, 1217-1240.

Metz JHM 1975. Time patterns of feeding and rumination in domestic cattle. $\mathrm{PhD}$ thesis, University of Wageningen, Wageningen, The Netherlands.

Nydegger F, Gygax L and Egli W 2011. Automatisches Messer der Kaubewegungen bei Wiederkäuern mit Hilfe eines Drucksensors. Agrarforschung Schweiz 2, 60-65.

Peiretti PG, Meineri G, Miraglia N, Mucciarelli M and Bergero D 2006. Intake and apparent digestibility of hay or hay plus concentrate diets determined in horses by the total collection of feces and $n$-alkanes as internal markers. Livestock Science 100, 189-194.

Penning PD and Rutter SM 2004. Ingestive behaviour. In Herbage intake handbook (ed. PD Penning), pp. 151-175. The British Grassland Society, Reading, UK.

Piccand V, Cutullic E, Meier S, Schori F, Kunz PL, Roche JR and Thomet P 2013. Production and reproduction of Fleckvieh, Brown Swiss, and 2 strains of Holstein-Friesian cows in a pasture-based, seasonal-calving dairy system. Journal of Dairy Science 96, 5352-5363.

Prendiville R, Lewis E, Pierce KM and Buckley F 2010. Comparative grazing behavior of lactating Holstein-Friesian, Jersey, and Jersey $x$ Holstein-Friesian dairy cows and its association with intake capacity and production efficiency. Journal of Dairy Science 93, 764-774.

$\mathrm{R}$ Core Team 2012. R: a language and environment for statistical computing. $R$ Foundation for Statistical Computing, Vienna, Austria. Retrieved on 20 August 2015 from http://www.R-project.org/.

Reist $M$, Erdin D, von Euw D, Tschuemperlin $K$, Leuenberger $H$, Chilliard $Y$, Hammon HM, Morel C, Philipona C, Zbinden Y, Kuenzi N and Blum JW 2002. Estimation of energy balance at the individual and herd level using blood and milk traits in high-yielding dairy cows. Journal of Dairy Science 85, 3314-3327. Rook AJ 2000. Principles of foraging and grazing behaviour. In Grass - its production \& utilization (3rd edition) (ed. A Hopkins), pp. 229-241. Blackwell Science, Oxford, UK. 
Schori F and Münger A 2014. Intake, feed conversion efficiency and grazing behaviour of two Holstein cow strains in a pasture-based production system under organic farming in Switzerland. Organic Agriculture 4, 175-186.

Sehested J, Kristensen T and Søegaard K 2003. Effect of concentrate supplementation level on production, health and efficiency in an organic dairy herd. Livestock Production Science 80, 153-165.

Spann B, Diepolder M, Schmidtlein EM, Tusch S, Hermüheim A and Spengel D 2007. Umstellung zur ökologischen Milchviehhaltung - Vergleich verschiedene Leistungsparameter. In Angewandte Forschung und Beratung für den ökologischen Landbau in Bayern (volume 3) (ed. K Wiesinger), pp. 65-77. Schriftenreihe der Bayerischen Landesanstalt für Landwirtschaft, Freising, Germany.

Templeman RJ 2004. Experimental design and statistical methods for classical and bioequivalence hypothesis testing with an application to dairy nutrition studies. Journal of Animal Science 82, E162-E172.
Thanner S, Schori F, Bruckmaier RM and Dohme-Meier F 2014. Grazing behaviour, physical activity and metabolic profile of two Holstein strains in an organic grazing system. Journal of Animal Physiology and Animal Nutrition 98, 1143-1153.

Verband Deutscher Landwirtschaftlicher Untersuchungs- und Forschungsanstalten 1993. Handbuch der Landwirtschaftlichen Versuchs- und Untersuchungsmethodik (VDLUFA-Methodenbuch), Bd. III: Die Chemische Untersuchung von Futtermitteln. VDLUFA-Verlag, Darmstadt, Germany.

Vicari T, van den Borne JJGC, Gerrits WJJ, Zbinden Y and Blum JW 2008. Postprandial blood hormone and metabolite concentrations influenced by feeding frequency and feeding level in veal calves. Domestic Animal Endocrinology 34, 74-88.

Washburn SP and Mullen KAE 2014. Invited review: genetic considerations for various pasture-based dairy systems. Journal of Dairy Science 97, 5923-5938. 